\title{
Problems and Barriers Affecting Total Productive Maintenance Implementation
}

\author{
Muhammad Adeel Munir \\ Department of Mechanical, \\ Manufacturing and Mechatronics \\ Engineering, University of Engineering \\ \& Technology, Lahore, Pakistan \\ adeelmunir158@gmail.com
}

\author{
Muhammad Zeeshan Rafique \\ Department of Mechanical Engineering, \\ Faculty of Engineering \& Technology, \\ University of Lahore, Lahore, Pakistan \\ muhammadzeeshanrafique@gmail.com
}

\author{
Mansoor Ali Zaheer \\ Department of Mechanical Engineering, \\ The University of Sargodha, \\ Sargodha, Pakistan
mechengineer778@gmail.com \\ Sargodha, Pakistan
mechengineer778@gmail.com
}

\section{Muhammad Anas Rasool}

Department of Mechanical Engineering, Faculty of Engineering \& Technology, University of Lahore, Lahore, Pakistan muhammad.anas@me.uol.edu.pk

\begin{abstract}
Mustafa Haider
Department of Mechanical Engineering, Faculty of Engineering \& Technology, University of Lahore, Lahore, Pakistan mustafah.haider@me.uol.edu.pk
\end{abstract}

\author{
Muhammad Saad Amjad \\ Department of Mechanical Engineering, \\ Faculty of Engineering \& Technology, \\ University of Lahore, Lahore, Pakistan \\ saadamjad95@gmail.com
}

\begin{abstract}
Total productive maintenance (TPM) is at the forefront of the maintenance strategies which brought about a paradigm shift from repair-maintenance strategy to proactive maintenance. The philosophy of TPM entails the facets of cost reduction and increased productivity. However, it has been observed that many manufacturing enterprises have made unsuccessful attempts at its adoption. In order to detect and overcome a qualitative research methodology has been employed and a survey has been carried out identifying the barriers and categorizing them to strategic, managerial, departmental, financial, and social regimes through rigorous data analysis. It was observed that strategic constraints are primarily detrimental to the success of TPM due to the lack of defined strategies for workers by management. Moreover, the departmental constraints are present due to misalignment between TPM and departmental objectives, in addition to the lack of training for workers in the system for TPM implementation. Moreover, there are managerial and financial constraints due to the lack of information visibility and finances, social constraints due to gap between top management authorization and worker mentality. Finally, a proper strategy has been proposed to address the issues affecting TPM implementation.
\end{abstract}

Keywords-total productive maintenance; Cronbach's alpha; correlation analysis; one way ANOVA

\section{INTRODUCTION}

Total productive maintenance (TPM) is a technique employed to improve the performance of a plant and its assets. For its successful implementation, organizations require unequivocal top management support and commitment, whereas employees should be highly motivated and empowered. Also, there should be a common conception among top management that TPM is a gradual process that demands patience and determination to achieve positive results. Due to the new production technology and techniques, the competition between different companies is increasing every day. The emphasis is not only to gain maximum production but also product quality. The main purpose of TPM technique is to develop coordination between maintenance and production departments for continuously improving the manufacturing process. The main philosophy of TPM is no breakdown of machines, which ultimately leads to minimization of the production loss, whilst efficiently using human resources. It is a common narrative that the best quality machines produce the best quality products. The production department should take responsibility of seemingly trivial tasks as lubrication of machines, cleaning of the work area and machines, followed by regular checkups. Machine operators should be motivated and empowered for small tasks, while the maintenance department should give full attention to the improvement of machinery and all the tasks that require skilled human resources. TPM was incepted by a Japanese organization Nippondenso, a subsidiary of Toyota Motors that introduced the concept of autonomous maintenance which implies that performing daily maintenance tasks is a responsibility of the operators. This helped in saving a lot of time and reaping economic rewards. The maintenance department was mandated to improve the overall equipment effectiveness (OEE) by using TPM. The common benefits of TPM include employee involvement, performance improvement and quality production. By making an operator the stakeholder of maintenance tasks, the skill level increases which results in positive social performance.

There are several factors that play an important role in successful implementation of TPM. Ground realities for each country should be taken into context while its implementation, keeping in mind that TPM is a gradual process. There should be a structured training for TPM coordinators and team members aided by financial resources. A system that measures performance and economic benefits should be developed. The 
involvement of top management and their communication with all levels of employees is an essential requirement for TPM implementation. Firstly, managers have to establish a consolidated strategy for the effective utilization of all employees. There should be clear policies and plans for the implementation of TPM in a company. Higher management should establish a separate department in which a dedicated manager could control the process and evaluate performance. The successful TPM implementation may include cultural change, alignment of company's goals with TPM goals, provision of appropriate financial resources for TPM, high level of cooperation among production and maintenance department, proper training and skill improvement, and removal of the barriers for its implementation. The success of TPM is highly dependent on the effectiveness of employees, so multi-skilled employee training is of paramount importance, which should not be confined to developing technical expertise but stretches to character and behavior improvement. Through proper training and effective utilization of employees, higher productivity and high quality standards can be met as the defects will be minimized and the breakdown frequency will be reduced by reducing the defects and breakdowns. Rewards and compensation in salary should be provided based on the success in the implementation of TPM. The results of employee's evaluation should be shared with them so they can be motivated. The performance should be improved by focus improvements and there should be a proper system to get feedback from the employees in order to be able to overall improve the system.

\section{LITERATURE REVIEW}

Many years ago, Japanese companies revealed that the performance of machines can not only be improved by the system but also by the people working on that machine. The term TPM was first time used by Nippondenso. They first time discussed getting more output by using the employees effectively. Later, Nakajima implemented TPM in Japanese plants. The main idea was that the basic responsibilities of the maintenance team should be given to operators. Operators have more knowledge about machines, and they can continuously improve them by extensive involvement and autonomy. The intensity of barriers in implementation of TPM was evaluated through graph theoretic approach [1]. The barriers for successful implementation of TPM were identified. Barriers may differ from organization to organization. They were grouped into categories that were behavioral barriers, human and cultural barriers, strategic barriers, operational barriers and technical barriers. Behavioral barriers were found to be the most effective and intense. This category includes top management failure, lack of vision, fear of job security, and poor cooperation between maintenance and production department. Quality of products and program cannot be increased without the involvement of top management which involves operational barriers, including the lack of standard operating procedures, improper maintenance schedule, bad working conditions and lack of performance measurement. Human and cultural barriers entail the lack of motivation, recognition and empowerment. Technical barriers can be handled by providing quality training for all employees.
TPM and how it is utilized in manufacturing sector was studied in [2]. Industries were categorized based on their region and type and the tools used for the implementation of TPM were analyzed. Maintenance is considered as the strategy for success [3]. There should be a reliable maintenance system and managers should consider the importance of equipment efficiency and reliability. The quality of products depends on the condition of the machines and quality leads the competition in a global scale. There is high competition, so the maintenance system should be considered as an integral part of the company. Teamwork in maintenance leads to the success of the industry [4]. The TPM is a technology-oriented concept and its failures are due to organizational problems, the style of management, lack of coordination between production and maintenance, and the lack of autonomy of the team which implements the TPM. Firm competitiveness depends upon how efficiently is managed in its resources and culture, and how its objectives are gained for better achievements [5]. Due to globalization and open trade, the firms are working in a highly competitive environment. By using competitive maintenance techniques, medium and small sized industries reached global competition. Business improvement occurs due to the implementation of TPM and total quality management (TQM) in manufacturing sector [6].

In [7], it was found that the success of maintenance techniques can be achieved by the involvement of employees, cultural change, and continuous improvement. TPM and TQM are basic maintenance techniques for improvement and growth in business. If companies adopt preventive maintenance approach, it will reduce the costs from $2 \%$ to $16 \%$, but there is a prevalent culture of parts' replacement after they are worn out. Due to downtime of machines, the expenditure of companies' increases but the losses due to human laziness are more than the losses due to downtime. This could be improved by effective use of human power, continuous training and change of culture. The factors which effect the successful implementation of TPM in Indian manufacturing companies were examined in [8]. It was found that the basic problems in the implementation of TPM were insufficient management support and lack of training. Companies implemented the program and did not give time for performance measurement. There were no properly defined objectives for the TPM. TPM objectives must be aligned with the business plan because TPM is holistic in nature, its objectives are not limited to production only. In Indian manufacturing industry, maintenance is treated as needless and generally, no attention is paid to it. It is considered as a reactive problem which has expenses that cannot be minimized, since rework is considered a part of the manufacturing process. Lack of motivation, rigid unions, inconsistency, and absence of planned maintenance are key factors for the failure of TPM. There was no recognition, no reward, and a lack of trust from the maintenance department to the production regarding their ability to take the responsibility of small maintenance tasks. An efficient TPM program can solve all the maintenance related problems. Many companies achieved improvements in their business by using the TPM approach. The issues related to the maintenance which effect the improvements in organization and create barriers at the initial stage of TPM were recognized in [9]. It was observed 
that TPM implementation improved the system by increasing productivity and quality, and decreasing cost. TPM avoids the equipment related losses and continuously improves performance. When the performance of the equipment is optimized, there will be fewer breakdowns and better employee performance. TPM improves the relation between maintenance and other departments of the manufacturing industry, which improves the reliability and at the end OEE.

There are many challenges which are raised at the initial stage of TPM implementation [10]. The performance measurement system was divided into three stages. The first stage was the design to measure the performance, the second was its implementation and the third its analysis by using feedback. Without the full commitment of employees, no issues can be solved. OEE only cannot measure the performance and effects TPM because it measures the internal factors and ignores external performance. To measure the performance improvement, both internal and external factors should be considered. External factors include the satisfaction of customers, on time delivery, after sales service and quality of products. The maintenance performance measurement (MPM) model was devised which measured losses due to poor equipment performance. The health and safety system was also considered. The only support between the planning and execution of maintenance is the availability of spare parts, tools and manuals which increase performance. One of the most important facets is the trained personnel. Unskilled personnel act as a demotivating factor. It was observed that when the problem in any maintenance system arises, the main issue is its reporting. There should be proper and planned structure for its reporting and how to solve it. MPM measures all the factors and provides proper estimation for the maintenance part in business. The philosophy of TPM was used in timber companies in the initiation and implementation of the maintenance of machines before breakdown occurs, by using quality training and improvement of the process [11]. Problems and complexities are found when one tries to change the culture and employees' involvement in the process. Management should adopt a participative way and should keep in mind that TPM will take long time to bring its benefits. Despite the difficulties in implementing the program, this program is necessary in the competitive market. All the departments including top management, maintenance, and production will have to participate and facilitate PM implementation. Participation of all personnel is a key factor in the implementation of TPM. The commitment and involvement of workers is used to align the objectives of TPM with the organizational objectives. For any type of improvements, there should be a search for all the areas which require improvement. There should be a proper structured team which must be able to find all those areas and they should actively participate to identify the problems and their solutions. The employees should be given responsibilities of their own interest.

When there is an implementation program in any company, researchers should observe the process and find the difference between the academic and the actual process. Standard operating procedures may be created, keeping in mind the ground realities. The main reason for failure of TPM is the lack of coordination between production and maintenance [12]. In
[12], industries were divided in three levels. The ones that claim to have implemented TPM and carry the structure to implement it, the ones that claim that they implemented TPM, but they have not the necessary structure, and the ones who have implemented it, but just to satisfy audits. Managers and administration who do not understand the real philosophy of TPM are the main reasons for its failure. Audits show that they have implemented everything positively but there is not actual improvement in the process and OEE. There is a negative attitude by companies including the decrease of budget for TPM by considering it as waste of time, selection of incompetent personnel, unscheduled maintenance, lack of commitment, etc. There are two types of maintenance. Time based maintenance (TBM) and condition based maintenance (CBM) [13]. CBM is more realistic than TBM but there is a need for improvement in CBM. The CBM uses the fact that the $99 \%$ of failures can be predicted by machine and equipment condition.

\section{RESEARCH METHODOLOGY}

A questionnaire was designed with five possible answers for each statement: strongly agree, agree, neutral, disagree and strongly disagree. The questionnaire was distributed among the people with different work positions, e.g. managers, assistant managers, supervisors, and workers in 3 different companies. The questionnaire consisted of 27 questions which covered seven basic factors which cause the failure of TPM. The factors (strategically constraints, managerial constraints, departmental constraints, financial constraints, social constraints, insufficient training, and lack of workers' commitment and lack of workers' commitment) were given numbers from 1 to 7 respectively (Table I). The questionnaire was distributed in three different companies labeled as Companies "A", "B", "C". A total of 93 responses were returned, 9 responses from managers, 26 from assistant managers, 18 from supervisors, and 39 responses from workers. They showed that the strategic issue is the most effective regarding the failure of TPM, followed by departmental, insufficient training, managerial, financial, and social and lack of workers commitment.

TABLE I. FAILURE FACTOR NUMBERING

\begin{tabular}{|c|c|}
\hline Factor & Number \\
\hline Strategic constraints & 1 \\
\hline Managerial constraints & 2 \\
\hline Departmental constraints & 3 \\
\hline Financial constraints & 4 \\
\hline Social constraints & 5 \\
\hline Insufficient training & 6 \\
\hline Lack of workers' commitment & 7 \\
\hline
\end{tabular}

\section{RESUlTS}

In order to check the reliability of data, the Statistical Package for the Social Sciences (SPSS) was used to ensure that no falsification took place while filling the questionnaire, employing Cronbach's alpha. If the significance value is less than 0.05 then both factors have different means and their effect on TPM is different. If their value is more than 0.05 then they have the same mean and their effect on failure of TPM is the same. Post hoc analysis was performed in SPSS. The value 
for Company A, B, and C was found to be $0.713,0.680$, and 0.75 respectively. The overall value for Cronbach's alpha was 0.79 which shows that the data were reliable (Table II). The ANOVA technique was performed in mathematical software to find the most effective factors that cause failure of TPM and the turkey test was performed. Table III shows the subsets. There are 3 subsets of factors and each subset has the same mean value and the same effect on TPM. In Figure 1, we can see that the strategic issue is the most effective in failure of TPM.

TABLE II. ANOVA FOR ALL FACTORS

\begin{tabular}{|c|c|c|c|c|c|c|}
\hline \multicolumn{2}{|c|}{ Factors } & \multirow{2}{*}{$\begin{array}{c}\text { Mean } \\
\text { difference } \\
\text { (I-J) }\end{array}$} & \multirow[b]{2}{*}{ Std. Error } & \multirow[b]{2}{*}{ Sig. } & \multicolumn{2}{|c|}{ 95\% Confidence interval } \\
\hline I & $\mathbf{J}$ & & & & $\begin{array}{l}\text { Lower } \\
\text { bound }\end{array}$ & $\begin{array}{l}\text { Upper } \\
\text { bound }\end{array}$ \\
\hline \multirow{6}{*}{1} & 2 & $-0.416489362^{*}$ & 0.090305867 & 0.000 & -0.68357675 & -0.14940198 \\
\hline & 3 & $-0.308510638^{*}$ & 0.090305867 & 0.012 & -0.57559802 & -0.04142325 \\
\hline & 4 & $-0.422872340^{*}$ & 0.090305867 & 0.000 & -0.68995973 & -0.15578495 \\
\hline & 5 & $-0.461879433^{*}$ & 0.090305867 & 0.000 & -0.72896682 & -0.19479205 \\
\hline & 6 & $-0.331382979^{*}$ & 0.090305867 & 0.005 & -0.59847037 & -0.06429559 \\
\hline & 7 & $-0.610815599^{*}$ & 0.090305867 & 0.000 & -0.87790299 & -0.34372821 \\
\hline \multirow{6}{*}{2} & 1 & $0.416489362^{*}$ & 0.090305867 & 0.000 & 0.14940198 & 0.68357675 \\
\hline & 3 & 0.107978723 & 0.090305867 & 0.896 & -0.15910866 & 0.37506611 \\
\hline & 4 & -0.006382979 & 0.090305867 & 1.000 & -0.27347037 & 0.26070441 \\
\hline & 5 & -0.045390071 & 0.090305867 & 0.999 & -0.31247746 & 0.22169732 \\
\hline & 6 & 0.085106383 & 0.090305867 & 0.965 & -0.18198100 & 0.35219377 \\
\hline & 7 & -0.194326238 & 0.090305867 & 0.324 & -0.46141362 & 0.07276115 \\
\hline \multirow{6}{*}{3} & 1 & $0.308510638^{*}$ & 0.090305867 & 0.012 & 0.04142325 & 0.57559802 \\
\hline & 2 & -0.107978723 & 0.090305867 & 0.896 & -0.37506611 & 0.15910866 \\
\hline & 4 & & 0.090305867 & 0.867 & -0.38144909 & 0.15272568 \\
\hline & 5 & -0.153368794 & 0.090305867 & 0.617 & -0.42045618 & 0.11371859 \\
\hline & 6 & -0.022872340 & 0.090305867 & 1.000 & -0.28995973 & 0.24421505 \\
\hline & 7 & $-0.302304961^{*}$ & 0.090305867 & 0.015 & -0.56939235 & -0.03521757 \\
\hline \multirow{6}{*}{4} & 1 & $0.422872340^{*}$ & 0.090305867 & 0.000 & 0.15578495 & 0.68995973 \\
\hline & 2 & 0.006382979 & 0.090305867 & 1.000 & -0.26070441 & 0.27347037 \\
\hline & 3 & 0.114361702 & 0.090305867 & 0.867 & -0.15272568 & 0.38144909 \\
\hline & 5 & -0.039007092 & 0.090305867 & 1.000 & -0.30609448 & 0.22808029 \\
\hline & 6 & 0.091489362 & 0.090305867 & 0.951 & -0.17559802 & 0.35857675 \\
\hline & 7 & -0.187943259 & 0.090305867 & 0.365 & -0.45503065 & 0.07914413 \\
\hline \multirow{6}{*}{5} & 1 & $0.461879433^{*}$ & 0.090305867 & 0.000 & 0.19479205 & 0.72896682 \\
\hline & 2 & 0.045390071 & 0.090305867 & 0.999 & -0.22169732 & 0.31247746 \\
\hline & 3 & 0.153368794 & 0.090305867 & 0.617 & -0.11371859 & 0.42045618 \\
\hline & 4 & 0.039007092 & 0.090305867 & 1.000 & -0.22808029 & 0.30609448 \\
\hline & 6 & 0.130496454 & 0.090305867 & 0.777 & -0.13659093 & 0.39758384 \\
\hline & 7 & -0.148936167 & 0.090305867 & 0.650 & -0.41602355 & 0.11815122 \\
\hline \multirow{6}{*}{6} & 1 & $0.331382979^{*}$ & 0.090305867 & 0.005 & 0.06429559 & 0.59847037 \\
\hline & 2 & -0.085106383 & 0.090305867 & 0.965 & -0.35219377 & 0.18198100 \\
\hline & 3 & & 0.090305867 & 1.000 & -0.24421505 & 0.28995973 \\
\hline & 4 & -0.091489362 & 0.090305867 & 0.951 & -0.35857675 & 0.17559802 \\
\hline & 5 & -0.130496454 & 0.090305867 & 0.777 & -0.39758384 & 0.13659093 \\
\hline & 7 & $-0.279432621^{*}$ & 0.090305867 & 0.033 & -0.54652001 & -0.01234523 \\
\hline \multirow{6}{*}{7} & 1 & $0.610815599^{*}$ & 0.090305867 & 0.000 & 0.34372821 & 0.87790299 \\
\hline & 2 & 0.194326238 & 0.090305867 & 0.324 & -0.07276115 & 0.46141362 \\
\hline & 3 & $0.302304961^{*}$ & 0.090305867 & 0.015 & 0.03521757 & 0.56939235 \\
\hline & 4 & 0.187943259 & 0.090305867 & 0.365 & -0.07914413 & 0.45503065 \\
\hline & 5 & 0.148936167 & 0.090305867 & 0.650 & -0.11815122 & 0.41602355 \\
\hline & 6 & $0.279432621^{*}$ & 0.090305867 & 0.033 & 0.01234523 & 0.54652001 \\
\hline
\end{tabular}

A comparison was made between the people of different posts such as managers, assistant managers, supervisors and workers. We see from Table IV that the significance value of insufficient training is greater than 0.05 so all people from all designations agree that insufficient training is a basic cause for the failure of TPM. The second factor on which all people agree is the financial factor, with a value of 0.05 . Moreover, correlation defines the effect of one variable on another. Table $\mathrm{V}$ shows that the most effective relation is between the social issues and the lack of workers' commitment, having a value of 0.616 .

TABLE III. POST HOC ANALYSIS IS SPSS

\begin{tabular}{|c|c|c|c|c|}
\hline \multirow{2}{*}{ Factors } & \multirow{2}{*}{$\mathbf{N}$} & \multicolumn{3}{|c|}{ Subset for alpha $=\mathbf{0 . 0 5}$} \\
\hline & & 1 & 2 & 3 \\
\hline 1 & 94 & 1.78989362 & & \\
\hline 3 & 94 & & 2.09840426 & \\
\hline 6 & 94 & & 2.12127660 & \\
\hline 2 & 94 & & 2.20638298 & 2.20638298 \\
\hline 4 & 94 & & 2.21276596 & 2.21276596 \\
\hline 5 & 94 & & 2.25177305 & 2.25177305 \\
\hline 7 & 94 & & & 2.40070922 \\
\hline Sig. & & 1.000 & 0.617 & 0.324 \\
\hline
\end{tabular}

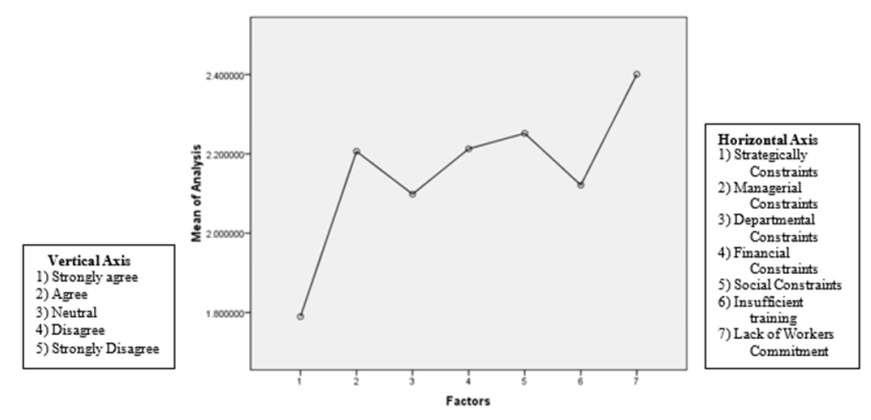

Fig. 1. Ordering of all factors

TABLE IV. COMPARISON BETWEEEN PEOPLE OF DIFFERENT DESIGNATIONS

\begin{tabular}{|c|c|c|c|c|c|c|}
\hline & & $\begin{array}{l}\text { Sum of } \\
\text { squares }\end{array}$ & $\begin{array}{c}\text { Degree } \\
\text { of }\end{array}$ & $\begin{array}{c}\text { Mean } \\
\text { square }\end{array}$ & $\mathbf{F}$ & Sig. \\
\hline \multirow{3}{*}{1} & Between groups & 4.456 & 3 & 1.485 & 7.703 & 0.000 \\
\hline & Within groups & 17.162 & 89 & 0.193 & & \\
\hline & Total & 21.618 & 92 & & & \\
\hline \multirow{3}{*}{2} & Between groups & 12.634 & 3 & 4.211 & 35.079 & 0.000 \\
\hline & Within groups & 10.685 & 89 & 0.120 & & \\
\hline & Total & 23.320 & 92 & & & \\
\hline \multirow{3}{*}{3} & Between groups & 4.071 & 3 & 1.357 & 7.605 & 0.000 \\
\hline & Within groups & 15.881 & 89 & 0.178 & & \\
\hline & Total & 19.952 & 92 & & & \\
\hline \multirow{3}{*}{4} & Between groups & 8.489 & 3 & 2.830 & 4.637 & 0.005 \\
\hline & Within groups & 54.307 & 89 & 0.610 & & \\
\hline & Total & 62.796 & 92 & & & \\
\hline \multirow{3}{*}{5} & Between groups & 13.817 & 3 & 4.606 & 10.187 & 0.000 \\
\hline & Within groups & 40.236 & 89 & 0.452 & & \\
\hline & Total & 54.053 & 92 & & & \\
\hline \multirow{3}{*}{6} & Between groups & 1.253 & 3 & 0.418 & 1.851 & 0.144 \\
\hline & Within groups & 20.076 & 89 & 0.226 & & \\
\hline & Total & 21.329 & 92 & & & \\
\hline \multirow{3}{*}{7} & Between groups & 8.836 & 3 & 2.945 & 7.519 & 0.000 \\
\hline & Within groups & 34.865 & 89 & 0.392 & & \\
\hline & Total & 43.701 & 92 & & & \\
\hline
\end{tabular}

It is a general perception that workers resist change. Worker unions resist change as they think that TPM will give more burdens to the workers so there is a strong correlation between the workers and social issues, such as job security, 
difficulty in working, complicated working conditions, intricate processes, etc. Table $\mathrm{V}$ shows the strong relationship between strategic and social issues.

TABLE V. CORRELATION ANALYSIS

\begin{tabular}{|c|c|c|c|c|c|c|c|c|}
\hline \multicolumn{2}{|c|}{ Factors } & 1 & 2 & 3 & 4 & 5 & 6 & 7 \\
\hline \multirow{3}{*}{1} & $\mathbf{P}$ & 1 & $0.549 * *$ & $0.441 * *$ & $0.255^{*}$ & $0.572 * *$ & 0.152 & $0.421 * *$ \\
\hline & $\mathbf{S}$ & & 0.000 & 0.000 & 0.013 & 0.000 & 0.145 & 0.000 \\
\hline & $\mathbf{N}$ & 94 & 94 & 94 & 94 & 94 & 94 & 94 \\
\hline \multirow{3}{*}{2} & $\mathbf{P}$ & $0.549 * *$ & 1 & $0.380 * *$ & $0.467 * *$ & $0.501 * *$ & 0.202 & $0.418 * *$ \\
\hline & $\mathbf{S}$ & 0.000 & & 0.000 & 0.000 & 0.000 & 0.051 & 0.000 \\
\hline & $\mathbf{N}$ & 94 & 94 & 94 & 94 & 94 & 94 & 94 \\
\hline \multirow{3}{*}{3} & $\mathbf{P}$ & $0.441 * *$ & $0.380^{* *}$ & 1 & 0.061 & $0.419 * *$ & 0.035 & $0.296 * *$ \\
\hline & $\mathbf{S}$ & 0.000 & 0.000 & & 0.561 & 0.000 & 0.739 & 0.004 \\
\hline & $\mathbf{N}$ & 94 & 94 & 94 & 94 & 94 & 94 & 94 \\
\hline \multirow{3}{*}{4} & $\mathbf{P}$ & $0.255^{*}$ & $0.467 * *$ & 0.061 & 1 & $0.361 * *$ & $0.497 * *$ & $0.391 * *$ \\
\hline & $\mathbf{S}$ & 0.013 & 0.000 & 0.561 & & 0.000 & 0.000 & 0.000 \\
\hline & $\mathbf{N}$ & 94 & 94 & 94 & 94 & 94 & 94 & 94 \\
\hline \multirow{3}{*}{5} & $\mathbf{P}$ & $0.572 * *$ & $0.501 * *$ & $0.419 * *$ & $0.361 * *$ & 1 & 0.310 ** & $0.616^{* * *}$ \\
\hline & $\mathbf{S}$ & 0.000 & 0.000 & 0.000 & 0.000 & & 0.002 & 0.000 \\
\hline & $\mathbf{N}$ & 94 & 94 & 94 & 94 & 94 & 94 & 94 \\
\hline \multirow{3}{*}{6} & $\mathbf{P}$ & 0.152 & 0.202 & 0.035 & $0.497 * *$ & $0.310^{* *}$ & 1 & 0.202 \\
\hline & $\mathbf{S}$ & 0.145 & 0.051 & 0.739 & 0.000 & 0.002 & & 0.051 \\
\hline & $\mathbf{N}$ & 94 & 94 & 94 & 94 & 94 & 94 & 94 \\
\hline \multirow{3}{*}{7} & $\mathbf{P}$ & $0.421 * *$ & $0.418^{* *}$ & $0.296^{* *}$ & $0.391 * *$ & $0.616^{* *}$ & 0.202 & 1 \\
\hline & $\mathbf{S}$ & 0.000 & 0.000 & 0.004 & 0.000 & 0.000 & 0.051 & \\
\hline & $\mathbf{N}$ & 94 & 94 & 94 & 94 & 94 & 94 & 94 \\
\hline
\end{tabular}

Strategically operators and supervisors should be empowered but there is a lack of trust between the upper management and supervisors and there is no proper two-way communication between them, so we can conclude that due to social barriers, the strategy to implement TPM can fail. The correlation between strategic and managerial issues can be explained in that way for the implementation of TPM. Its value is 0.549 . There should be dedicated managers to implement policies, strategies, and goals. In the absence of dedicated managers, there will be a lack of strategy that will lead to failure of TPM implementation in the company. Similarly, the value of correlation between managerial and social issues is 0.501 . If the management is effective, they will develop a strategy that will decrease social barriers. Moreover, they will work to form proper communication channels between workers and higher management which will build mutual understanding and trust between them. Another relationship exists between the financial constraints and insufficient training. Its value is 0.497. Due to financial constraints, proper education and training including information technology and experienced work force are not available.

\section{DISCUSSION}

TPM is a gradual and continuous improvement process which involves all employees and departments and the entire upper, middle and lower level management. Its main purpose is to increase machine productivity and administration efficiency. Most organizations usually spend their resources in repairs of machinery and after breakdowns. TPM focuses on prescheduling maintenance and preventive measures to save time and money. Top management is responsible to create favorable environment to implement TPM. They have to bring change in the mindset of employees. The machine operators should be empowered and they should do basic maintenance repairs. The responsibilities of operators should include cleaning and small maintenance tasks. Financial resources are key factors for the successful implementation of TPM. The benefits of TPM are obtained after a long time, so top level management should be patient. If the production department takes the responsibility of simple maintenance tasks, then the maintenance team will find more time to improve the system. Employees must be rewarded for their good performance. TPM includes higher productivity, low cost, high quality of products, safety in all factory areas, healthy relations between employees and their work. The training for all employees should be provided at an initial stage. New skills about TPM are an important part for its successful implementation. Statistical techniques can boost up the process towards success. TPM coordinators will provide training to management and shop floor employees. Support for TPM coordinators from the top management (including financial resources and involvement in process) will guarantee their success. A clear plan and strategy should be defined. An inside analysis of the company should be conducted including business details, financial condition, and strength. Weak areas should be analyzed preferably via this procedure rather than to depend on external analysis of other companies. The first step is to form a highly skilled team which should be highly educated and would be responsible for the implementation of TPM. In order to increase the involvement of employees, there should be a structured communication system between management and employees. The most difficult step in TPM implementation is to bring change in workers' mindset, as employees usually resist change. They are hesitant to take the responsibility but in the case of TPM, they play a vital role. By providing clear objectives and by empowering the employees by providing incentives, they may take part in the growth of company.

\section{CONCLUSION}

TPM is an effective strategy for process improvement but there are many constraints and barriers affecting its implementation. The first constrain that affects the system is the strategic constraint that plays the most important part in TPM failure, because there are no proper goals, guidelines and strategies defined by the management for workers due to which some workers get overloaded and some are not properly involved, which leads to resistance against TPM. The second constrain was the departmental constraint because the objectives of a department were not aligned with the objectives of TPM e.g. the department demands 24-hour operational machines to get maximum production without considering preventive maintenance and proper cleaning which are essential parts of TPM. TPM demands proper structure and a team that is usually not provided by the department. The third constraint is insufficient training, agreed upon by all respondents. Management emphasizes on initial training but with the passage of time, they do not provide structured training, although TPM is a continuous process. Ground reality must be considered while providing training. The use of customized information technology in a proper way will boost success. Managerial constraint refers to the lack of communication between top management and employees. Top 
management wants to get immediate benefits without considering that TPM is a very long process, and it is incumbent upon them to provide healthy working environment. Financial constraints are another hurdle since the organization's top management is unsure about the Return On Investment (ROI) and Cost-To-Benefit Ratio (CBR) in TPM implementation. It is a widespread narrative that TPM will exert financial burdens and the production quantity will be affected. Last examined was the social constraint which is caused by the authoritarian behavior of management, due to which there is a gap between top management and workers. In the future, research can be conducted regarding the economic benefits of TPM and the related social impacts from worker's, management and organizational points of view.

\section{REFERENCES}

[1] R. Attri, S. Grover, N. Dev, "A Graph Theoretic Approach to Evaluate the Intensity of Barriers in the Implementation of Total Productive Maintenance (TPM)", International Journal of Production Research, Vol. 52, No. 10, pp. 3032-3051, 2014

[2] A. K. Digalwar, P. V. Nayagam, "Implementation of Total Productive Maintenance in Manufacturing Industries: A Literature-Based Metadata Analysis", IUP Journal of Operations Management, Vol. 13, No. 1, pp. $1-15,2014$

[3] I. S. Ahuja, "Exploring the Impact of Effectiveness of Total Productive Maintenance Strategies in Manufacturing Enterprise", International Journal of Productivity and Quality Management, Vol. 9, No. 4, pp. 486501,2012

[4] M. Rolfsen, C. Langeland, "Successful Maintenance Practice through Team Autonomy", Employee Relations, Vol. 34, No. 3, pp. 306-321, 2012

[5] G. Sardana, S. Sinha, "Enhancing Firm Competitiveness in Fast Track through Total Productive Maintenance", International Journal of Indian Culture and Business Management, Vol. 4, No. 1, pp. 88-103, 2010

[6] M. Kaur, K. Singh, I. Singh Ahuja, "An Evaluation of the Synergic Implementation of Tqm and Tpm Paradigms on Business Performance", International Journal of Productivity and Performance Management, Vol. 62, No. 1, pp. 66-84, 2012

[7] M. C. Eti, S. Ogaji, S. Probert, "Reducing the Cost of Preventive Maintenance (PM) through Adopting a Proactive Reliability-Focused Culture”, Applied Energy, Vol. 83, No. 11, pp. 1235-1248, 2006

[8] G. Singh, I. S. Ahuja, "Strategies and Success Factors for Overcoming Challenges in Jit Implementation in Indian Manufacturing Industry", International Journal of Technology, Policy and Management, Vol. 13, No. 1, pp. 15-33, 2013

[9] I. Ahuja, J. Khamba, "An Evaluation of TPM Implementation Initiatives in an Indian Manufacturing Enterprise", Journal of Quality in Maintenance Engineering, Vol. 13, No. 4, pp. 338-352, 2007

[10] A. Parida, U. Kumar, "Maintenance Performance Measurement (MPM): Issues and Challenges", Journal of Quality in Maintenance Engineering, Vol. 12, No. 3, pp. 239-251, 2006

[11] J. Garcia Arca, J. Carlos Prado Prado, "Personnel Participation as a Key Factor for Success in Maintenance Program Implementation: A Case Study", International Journal of Productivity and Performance Management, Vol. 57, No. 3, pp. 247-258, 2008

[12] M. Rodrigues, K. Hatakeyama, "Analysis of the Fall of TPM in Companies", Journal of Materials Processing Technology, Vol. 179, No. 1-3, pp. 276-279, 2006

[13] R. Ahmad, S. Kamaruddin, "An Overview of Time-Based and Condition-Based Maintenance in Industrial Application”, Computers \& Industrial Engineering,Vol. 63, No. 1, pp. 135-149, 2012 